\title{
Phase transformations at interfaces: Observations from atomistic modeling
}

\author{
T. Frolov ${ }^{1}$, M. Asta ${ }^{2}$ and Y. Mishin ${ }^{3}$ \\ September 16, 2017
}

${ }^{1}$ Lawrence Livermore National Laboratory, Livermore, California 94550, USA

${ }^{2}$ Department of Materials Science and Engineering, University of California, Berkeley, California 94720, USA

3 Department of Physics and Astronomy, MSN 3F3, George Mason University, Fairfax, Virginia 22030, USA

\begin{abstract}
We review the recent progress in theoretical understanding and atomistic computer simulations of phase transformations in materials interfaces, focusing on grain boundaries (GBs) in metallic systems. Recently developed simulation approaches enable the search and structural characterization of GB phases in single-component metals and binary alloys, calculation of thermodynamic properties of individual GB phases, and modeling of the effect of the GB phase transformations on GB kinetics. Atomistic simulations demonstrate that the GB transformations can be induced by varying the temperature, loading the GB with point defects, or varying the amount of solute segregation. The atomic-level understanding obtained from such simulations can provide input for further development of thermodynamics theories and continuous models of interface phase transformations while simultaneously serving as a testing ground for validation of theories and models. They can also help interpret and guide experimental work in this field.
\end{abstract}

Keywords: Molecular dynamics, Monte Carlo modeling, grain boundary phases, solute segregation, grain boundary migration, grain boundary diffusion.

\section{Introduction}

Recent years have seen a rapid growth of evidence suggesting that materials interfaces are capable of first-order structural transformations in which the interface properties (such as solute segregation, excess volume, mobility or sliding resistance) undergo discontinuous changes [1].

(C) 2016. This manuscript version is made available under the Elsevier user license http://www.elsevier.com/open-access/userlicense/1.0/ 
Experiments have revealed a potentially important role of grain boundary (GB) phase transitions (sometimes referred to as "complexion transitions" $[1,2]$ ) in abnormal grain growth in ceramics [2], activated sintering [3] and liquid metal embrittlement [4]. Layering transitions associated with GB segregation were investigated using lattice gas models [5, 6], first-principles calculations [7] as well as advanced electron microscopy and spectroscopy methods [8-10]. Experimental investigation of the potential impact of GB phase transitions on microstructure and other materials properties is currently a highly active area of research [1, 4, 11-13]. The experimental studies have raised a number of fundamental questions concerning the thermodynamic nature of the interface phases, their atomic structures and kinetic properties. A unified thermodynamic description of bulk and low-dimensional phases has been recently proposed [14], phase rules for phases of any dimensionality have been formulated, and adsorption equations for interface phases and line defects separating such phases have been derived [14]. Phase-field models have been developed [2, 15, 16], predicting a variety of possible interface transformations and mapping them onto bulk phase diagrams.

Although fundamentally important, the thermodynamic analysis [14, 17-19] and phasefield models $[2,15,16]$ do not provide atomic-level insights into interface phases and phase transformations. The goal of this paper is to review the recent progress in atomistic modeling of interface transformations, focusing on GBs in metallic systems as an important practical case. Although molecular dynamics (MD), Monte Carlo (MC) and other atomistic simulation methods have been successfully applied to study interfaces for a long time [20, 21], it has not been until very recently that it became possible to identify and structurally characterize individual GB phases, compute their thermodynamic properties, and observe transformations among them. We describe the recent work on structural transformations in single-component GBs (Sec. 2), in binary alloys (Sec. 3), and the effect of such transformations on GB migration and short-circuit diffusivity (Sec. 5). In Sec. 5 we discuss existing challenges in this field and outline future work.

\section{Grain boundary phase transitions in pure metals}

\subsection{The multiplicity of ground-state grain boundary structures}

Atomistic computer simulations have proven to be an invaluable tool for the study of GB properties [21]. Such simulations have been successfully applied to predict GB structures and calculate their thermodynamic and kinetic properties, such as GB free energies, diffusivities and mobilities as functions of temperature and chemical composition [21-30]. However, despite the extensive research, there has been no convincing simulation evidence of GB phase transformations until very recently (except for the dislocation-pairing transition in relatively low-angle GBs [31]). It has been demonstrated that the critical impediment to observations of such transformations was rooted in inadequate simulation methodology, namely, in fixing the total number of atoms and using periodic boundary conditions. In order to sample all possible atomic configurations, atoms need to be added to or removed from the GB region [32-36]. To overcome this shortcoming of the previous simulations, a new simulation methodology has been developed that allows for variations of the atomic density in the GB region as the latter approaches thermodynamic equilibrium [37]. Applications of this methodology have 
led to the discovery of multiple GB phases and phase transitions by varying the temperature and/or concentration of impurities or point defects [22, 37, 38].

The new simulation approach has been tested by studying the $\Sigma 5(210)$ and $\Sigma 5(310)$ symmetrical tilt GBs in several fcc metals modeled with embedded-atom method potentials. These are representative high-angle, high-energy boundaries that have been extensively investigated in the past. Their choice was additionally motivated by the recent experimental measurements of $\mathrm{Ag}$ radio-tracer diffusion in $\mathrm{Cu}$ bicrystals with the $25(310) \mathrm{GB}$ [39], which revealed a peculiar temperature behavior of the GB diffusivity that suggested a possible structural transformation. To test this hypothesis, atomic structures of the two $\Sigma 5$ GBs were optimized with respect to both atomic density and rigid translations of the grains [37]. It was found that at $0 \mathrm{~K}$, the GB energy as a function of the GB density (measured as the fraction of atoms in (210) and (310) planes) exhibits several local minima representing different GB phases (Fig. 1(a)). In each boundary, one minimum corresponds to the long-known structure composed of kite-shape structural units obtained without addition or removal of atoms (Fig. 1(b,d)). Other minima are new and represent the filled-kite and split-kite GB structures (Fig. 1(c,e,f)). These structures are more complex and are either non-periodic or have a long period whose exact value remains unknown. These structures were obtained in simulation blocks with a large GB area and should be considered as approximants of the actual ground-state structures. It is possible that structures with even lower energies could be found in still larger simulation blocks. Remarkably, and contrary to the common belief, none of the tested interatomic potentials predicts the normal kite structure to be the lowest in energy for the $\Sigma 5(210) \mathrm{GB}$.

It should be noted that the phases found in the $\Sigma 5$ GBs are characterized by different excess properties, such as the excess volume and the interface stress [37]. These differences may result in long-range elastic strain fields around interface phase junctions in multi-phase states, which in turn can affect the coexistence of different GB phases and interact with dislocations and other elements of the microstructure.

\subsection{Temperature-induced grain boundary transitions}

Atomistic simulations have shown that GB phases can reversibly transform to each other with temperature [37]. Since such transformations require significant changes in atomic density in the GB region, the conventional simulation methodology with periodic boundary conditions and a fixed number of atoms cannot be applied. To enable automatic adjustment of the atomic density during the transformation, a different methodology has been proposed in which one or both edges of the GB terminates at an open surface (Fig. 2(a)). In this setup, provided the temperature is high enough, atoms can leave or enter the GB by diffusion to/from the surface. This effectively makes the GB an open thermodynamic system capable of exchanging atoms with a reservoir, a condition which naturally exists under experimental conditions.

As an example, Fig. 2(a) shows a $\mathrm{Cu}$ bicrystal with the $\Sigma 5(310)$ boundary annealed at 800 $\mathrm{K}\left(0.6 T_{m}, T_{m}=1327 \mathrm{~K}\right.$ being the melting temperature predicted by this potential) for tens of nanoseconds. The zoomed-in views reveal the original normal-kite GB structure on the left and a new, split-kite structure on the right. The latter nucleates at the surface and gradually propagates inside the bicrystal during the simulation. Its growth is enabled by the supply of 
extra atoms from the surface, a process which is kinetically controlled by GB diffusion. At a certain temperature, the two GB phases can coexist in thermodynamic equilibrium separated by a line defect representing a one-dimensional phase boundary between two two-dimensional phases. Knowledge of thermodynamic and topological properties of these unusual defects is important for the understanding of GB phase transitions (particularly, GB phase nucleation). These defects deserve a detailed study in the future. The observation of coexistence of GB phases with different densities separated by an atomic-width line defect confirms that the phase transformation is first order in character. Similar fully reversible temperature-induced phase transitions were found in the $\Sigma 5(210)$ boundary [37].

\subsection{Grain boundary transitions induced by point defects}

During GB phase transformations, the boundary absorbs or rejects a large amount of atoms. This suggests that a phase transformation can be induced by injection or removal of a suitable number of point defects at a fixed temperature. This was indeed confirmed by simulations in which a GB phase was initially created in a periodic supercell isolated from external sources or sinks of atoms as in Fig. 3(a). A number of vacancies or interstitial atoms was then introduced into the GB region and the system was re-equilibrated by an MD run. For example, 80 interstitial atoms (the number required for transformation to the split-kite phase) were introduced into the normal-kite structure of the $\Sigma 5(310)$ GB followed by an anneal at $800 \mathrm{~K}$. A possible outcome could be a uniform redistribution of the extra atoms in the initial GB structure. Instead, a first-order transformation to the split-kite phase was observed as illustrated in Fig. 3(c). This transformation was fully reversible: when the same number of vacancies was subsequently introduced into the split-kite phase, the latter transformed back to normal kites (Fig. 3(e)). As a result of this phase transformation cycle, the boundary returned to its initial state having annihilated a large number of point

defects. Analogous transformation cycles were observed upon loading of point defects into the $\Sigma 5(210)$ GB.

These simulations suggest that interfacial phase transformations can greatly increase the capacity of GBs to absorb non-equilibrium point defects. This finding may have important practical implications for materials operating under extreme conditions. For example, the radiation tolerance of many nuclear materials depends on the efficiency of GBs as sinks of vacancies and interstitials created during irradiation by energetic particles [40-43]. GB phase transformations present a novel mechanism of radiation damage healing in such materials.

\section{$3 \quad$ Segregation-induced grain boundary transitions}

In technological applications, dopants and impurities can play a dominant role in microstructure evolution and properties. Recent experimental studies of doped ceramics revealed discontinuous changes in coarsening behavior that were attributed to the emergence of GB phases [1, 2, 13]. Such phases are characterized by different segregated structures such as a monolayer, a bilayer, a trilayer, or a thick disordered layer [4, 12]. Recent atomistic simulations have provided insights in the thermodynamic origin and physical mechanisms responsible for phase transitions in segregated GBs [22, 38]. 


\subsection{Segregated grain boundary structures}

The choice of the $\Sigma 5(210)$ GB in Cu-rich Cu-Ag alloys as a model system to study segregation effects was prompted by the recent experimental measurements of $\mathrm{Ag}$ diffusion in this boundary [39] and the availability of a reliable interatomic potential reproducing the $\mathrm{Cu}-\mathrm{Ag}$ phase diagram [44]. The study combined MD with semi-grand canonical MC simulations. While MC simulations cannot capture the correct dynamics of phase changes, they allow one to quickly obtain the equilibrium distribution of chemical components over the system and compute equilibrium thermodynamic properties. This could not be achieved within the MD approach due to the long time scales associated with solid-state diffusion. In the semigrand canonical ensemble, the atoms are allowed to move away from equilibrium positions and simultaneously switch their chemical species while the temperature $T$, total number of atoms $N$ and the diffusion potential $M$ between $\mathrm{Ag}$ and $\mathrm{Cu}$ remain fixed.

Two different types of simulation block were employed. GB phase transitions were studied in a large bicrystal with a GB terminated at two open surfaces (Fig. 4(a)). Thermodynamic properties of individual GB phases were calculated in periodic simulation blocks containing a single GB phase of interest; due to the absence of sinks and sources of atoms, phase transformations in such blocks do not occur and properties of a single GB phase can be studied over and wide range chemical compositions and/or temperatures. A GB phase is characterized by a set of excess properties such as the excess energy, excess number of Ag atoms (segregation), interface stress and others. All these properties are readily accessible by atomistic simulations and served as input to thermodynamic integration of the adsorption equation to obtain the GB free energy $\gamma[22,38]$. As in the previous work [24], the excess quantities were calculated at a fixed total number of atoms using Cahn's formalism of generalized excesses [45].

Equilibrium segregated structures of the filled-kite and split-kite phases are illustrated in Fig. 4(b,c). The filled-kite phase exhibits a monolayer segregation whereas the split-kite phase a double layer segregation. The equilibrium segregation isotherms of the two phases at $900 \mathrm{~K}$ are shown in Fig. 4(d,f). It should be emphasized that the segregated layers are only enriched in but not fully packed with $\mathrm{Ag}$ atoms. In fact, the bilayer structure contains less Ag atoms per unit GB area than does the monolayer structure. Thus, caution should be exercised when interpreting experimental images of segregated GBs: the number of segregation layers is not a reliable indicator of segregation strength.

\subsection{Segregation effect on grain boundary transformations}

In pure $\mathrm{Cu}$, the transition between the split-kite and filled-kite phases of the $\Sigma 5(210)$ GB occurs at a temperature of about $1050 \mathrm{~K}$ [37]. The segregation isotherms indicate that at the temperature of $900 \mathrm{~K}$ (when the split-kite phase in pure $\mathrm{Cu}$ is more stable), Ag segregation into filled kites increases with the grain composition $c$ faster than does the segregation into split kites (Fig. 4(d,f)). From the Gibbs adsorption equation, we can conclude that the free energy $\gamma_{F K}$ of filled kites decreases with the grain composition faster than does the free energy $\gamma_{S K}$ of split kites. One can expect, therefore, that at some composition $c_{*}$, the two free energies become equal, $\gamma_{F K}=\gamma_{S K} \equiv \gamma_{*}$, and a segregation-induced phase transformation occurs. 
To test this prediction, a simulation block with two open surfaces was utilized (Fig. 4(a)). $\mathrm{MC}$ simulations were conducted at $900 \mathrm{~K}$ for a set of grain concentrations imposed by different diffusion potentials $M$. The boundary was initially composed of two phases separated by a phase junction. During the simulations, one of the phases always grew while the other shrunk, depending on the chosen $M$. A value $M=M_{*}$ was found at which the position of the phase junction fluctuated in both directions without any discernible growth of either phase. The respective grain composition, $c_{*}=0.02$ at.\% Ag, was identified with the GB phase equilibrium at this temperature. The phase change occurring at this composition is accompanied by a jump in Ag segregation marked by the vertical dashed line in Fig. 4(f). This calculation gives a point on the temperature-composition phase diagram shown in Fig. 4(g). Similar calculations at other temperatures would recover the entire phase coexistence line on this diagram.

The obtained phase coexistence point was used as a reference state for thermodynamic integration. Fig. 4(e) shows the free energies of the GB phases relative to this reference state, $\gamma_{F K}-\gamma_{*}$ and $\gamma_{S K}-\gamma_{*}$, as functions of the grain composition. Note that the free energy difference between the phases, $\gamma_{F K}-\gamma_{S K}$, is very small. Even when extrapolated to pure $\mathrm{Cu}$ at $900 \mathrm{~K}$, it reaches only $2.2 \mathrm{~mJ} / \mathrm{m}^{2}$. This is much smaller than typical GB energies in metals (e.g., $951 \mathrm{~mJ} / \mathrm{m}^{2}$ for this boundary at $0 \mathrm{~K}$ ). The free energy difference $\gamma_{F K}-\gamma_{S K}$ is the driving force for the GB phase transformation per unit area. Its small magnitude demonstrates that, applying the proposed simulation method, thermodynamic properties of individual GB phases can be computed with a high precision, including accurate location of GB phase transformation points. The ability to evaluate the driving force for GB transitions is crucial for the understanding of nucleation and growth of GB phases. For example, knowing the driving force and measuring the rate of GB phase growth, the mobility of the GB phase junction can be extracted. Kinetic properties of this novel line defect are presently unknown.

\section{Effect of grain boundary transformations on kinetic properties}

\subsection{Effect on grain boundary motion}

The recent experimental studies of the effect of GB transitions on grain growth kinetics $[1,13]$ utilized polycrystalline samples and could only provide information related to collective behavior and properties of multiple GBs. Atomistic simulations offer an effective tool for gaining deeper insights by examining the effect of specific, fully characterized, GB phases on kinetic properties of individual GBs. One of the approaches to the evaluation of GB kinetics is to drive a GB by an applied shear stress and extract its mobility from the velocityforce relation [46, 47]. The stress-driven GB motion is caused by the shear-coupling effect, in which normal displacements of a boundary are coupled to parallel translations of the grains [48-53]. The degree of coupling is characterized by the coupling coefficient $\beta=v_{\|} / v$, where $v_{\|}$is the grain translation velocity and $v$ the GB velocity. For ideal coupling, $\beta$ is a geometric factor that can be predicted from the GB crystallography. In the particular case of [001] symmetrical tilt GBs, there are two coupling modes with coupling factors $\beta_{\langle 100\rangle}=2 \tan (\theta / 2)$ and $\beta_{\langle 110\rangle}=-2 \tan (\pi / 4-\theta / 2)$, where $\theta$ is the misorientation angle. 
These two equations describe the $\langle 100\rangle$ and $\langle 110\rangle$ modes of coupling and were verified by both atomistic simulations [49-51] and experiments on Al bicrystals [53, 54].

The previous work on coupled GB motion of $\Sigma 5$ GBs was performed on normal-kite structural units and did not investigate other GB phases. The latter has been done recently using the $\mathrm{Cu} \Sigma 5(210)[001] \mathrm{GB}$ as a model. This boundary was previously found to couple according to the $\langle 110\rangle$ mode with a negative $\beta$ [48-53]. The split-kite and filled-kite phases of this boundary were created in simulation blocks that did not contain sinks or sources of atoms, which precluded phase transformations. A shear stress was applied to each boundary employing the previous methodology [49-51]. As illustrated in Fig. 5(a,b), under the same shear rate, the two GB phases move in opposite directions and with different speeds. To quantify this difference, the inverse coupling factors computed for the two phases are plotted as functions of temperature in Fig. 5(c). Until the temperature reaches about $0.83 T_{m}$, both phases move with ideal coupling factors but in different coupling modes: split kites in the $\langle 100\rangle$ mode $(\beta>0)$ while filled kites in the $\langle 110\rangle$ mode $(\beta<0)$. This behavior demonstrates that GB phase transformations can have a strong impact on GB migration. It suggests that in some cases, a phase transformation can even reverse the direction of GB motion by triggering a switch between different coupling modes. Thus, a possible indirect way to discover GB phase transitions is by measuring variations in the coupling factor at different temperatures and impurity levels.

At temperatures above $0.83 T_{m}$, the coupling factors of both GB phases become non-ideal. The motion of the filled-kite phase is accompanied by an increasing number of sliding events until the boundary completely pre-melts at $1300 \mathrm{~K}$ and its response to the applied shear switches to pure sliding $(\beta \rightarrow \infty)$. The split-kite phase also becomes increasingly disordered; its coupling factor becomes non-ideal and at about $1200 \mathrm{~K}$ changes sign. The shear stress required for sustaining the GB motion with a given velocity decreases with temperature (Fig. 5(d)). At high temperatures when the boundary becomes atomically disordered, the stress approaches zero. It is interesting to note that the stress required to move the filled-kite phase is about $60 \%$ of that for the split-kite phase. This observation suggests that, under a given driving force, a GB phase transition can significantly accelerate, slow down or even stop the GB motion.

\subsection{Effect on grain boundary diffusion}

GB diffusion is a structure-sensitive property. It is natural to expect that structural phase transformations in GBs can have a significant impact on GB diffusivity. Fig. 6 shows an Arrhenius plot, $\log P$ versus $1 / T$, of the calculated GB diffusion flux $P=D_{g b} \delta$ [37]. Here, $D_{g b}$ is the GB self-diffusion coefficient in the $\mathrm{Cu} \Sigma 5(310)[001] \mathrm{GB}$ and $\delta$ is the GB width. The calculations were performed in a periodic simulation block prohibiting GB phase transformations. The diffusion flux was computed from mean-square displacements of GB atoms during MD simulations [22]. Note that the split-kite phase is characterized by a smaller activation energy of diffusion in comparison with the normal-kite phase. The transition between the two phases occurs at approximately $800 \mathrm{~K}$, with the normal-kite phase being more stable below this temperature and the split-kite phase above. Fig. 6 suggests that the phase transition must be accompanied by a characteristic break in the Arrhenius dependence of $P$ at around $800 \mathrm{~K}$, with a lower activation energy at higher temperatures. This type of a 
break was indeed observed experimentally at about $800 \mathrm{~K}[39,55]$. This agreement strongly suggests that the unusual diffusion behavior found in experiments was indeed caused by the phase transformation in this boundary.

In a more recent study, Divinski and co authors [39] measured Ag impurity diffusivity in the $\mathrm{Cu} \Sigma 5(310) \mathrm{GB}$. The GB diffusivity was described by the diffusion flux $P=D_{g b} s \delta$, where $D_{g b}$ is the GB diffusion coefficient of $\mathrm{Ag}$ and $s$ is the impurity segregation factor. Similar to the self-diffusion case, a characteristic break in the Arrhenius dependence of $P$ was found at about $800-850 \mathrm{~K}$. To make a one to one comparison with the experimental findings, the same diffusion flux was computed for Ag diffusion in the individual kite and split-kite phases of this boundary by combining MD with MC simulations. The latter was applied to create the equilibrium GB segregation of $\mathrm{Ag}$ in each phase and to extract the segregation factor $s$ in the dilute regime. The results have revealed a break in the slope of the Arrhenius plot at about $800 \mathrm{~K}$ (Fig. 6), which is strikingly similar to the break observed in the experiment [39]. This close agreement provides additional confirmation that the non-Arrhenius diffusion behavior found by Divinski et al. [39] was caused by a GB phase transformation. The computed segregation factors were also in good agreement with experimental measurements [39], lending additional credence to the simulation results.

\section{Outlook}

The concept of interface phase transformations has been known since many years $[17,18,56$ 58]. In fact, the idea can be traced back to Gibbs [59]. The recent explosion of interest in the topic is primarily due to the appearance of new experimental evidence (although mostly indirect) for the existence of such transitions and their possible impact on materials properties. Significant progress has also been achieved in theoretical description of interface transitions $[14,17]$ and their modeling by continuous methods (such phase-field approaches [15, 16]) and atomistic simulations $[22,37,38,60]$. Many theoretical and computational challenges remain in this field. The current thermodynamic theory of interface phase transformations [14] relies on simplifying assumptions that make it applicable, strictly speaking, only to isotropic systems such as multicomponent fluids. The next challenge is to generalize the theory to interfaces between solid phases. Such interfaces are often anisotropic and their properties may depend on the crystallographic orientation of the interface plane. Furthermore, solid-solid interfaces possess two types of tension: the interface free energy and the interface stress, which are different quantities both conceptually and numerically [45, 59]. The theory also neglects the curvature effects on interface transformations. Incorporation of these effects would enable applications of the theory to a wider range of real materials.

One aspect that deserves special attention is related to the properties and role of the onedimensional "phase boundaries", i.e., junctions between interface phases. Understanding of these defects is crucial for developing theories of phase nucleation at interfaces and description of heterogeneous (multi-phase) states that may exist at interfaces under certain constraints. The recently proposed thermodynamic theory of such junctions should be extended to solidsolid interfaces, such as GBs (especially asymmetric type). In particular, the role of the elastic strain fields created by the phase junctions should be investigated and, if necessary, incorporated in the theory. 
On the computational side, the search for interface phases remains a difficult task even in the simplest case of single-component interfaces with a high symmetry. Clearly, the traditional approach to creating interfaces by simply joining two crystals and applying relative rigid translations and local atomic displacements is insufficient for this purpose [32$36,61,62]$. The recently proposed simulation methodologies $[22,37,38,60]$ create grandcanonical environments in which the atomic density in the interface regions becomes an additional automatically adjustable parameter leading to a deeper minimization of the interface free energy. However, such methodologies have certain limitations (e.g., the reliance on sufficiently fast GB diffusion) and are only implemented in specifically-designed simulation software. An important task for the future is the development of more robust grandcanonical methods and their implementation in commonly used MD and MC simulation packages. More efficient and accessible simulation approaches would enable computational discovery of new interface phases and deeper investigation of their junctions and multi-phase interface states. This would also provide a broader testing ground for the existing theories and inspiration for future new theories of interface phase transformations.

Acknowledgements. - This work was performed in part under the auspices of the U.S. Department of Energy by Lawrence Livermore National Laboratory under Contract DEAC52-07NA27344. Y.M. was supported by the National Science Foundation, Division of Materials Research, the Metals and Metallic Nanostructures Program. Use was made of computational resources provided under the Extreme Science and Engineering Discovery Environment (XSEDE), which is supported by the National Science Foundation under grant number OCI-1053575.

\section{References}

[1] P. R. Cantwell, M. Tang, S. J. Dillon, J. Luo, G. S. Rohrer, and M. P. Harmer, Acta Mater. 62, 1 (2013).

[2] S. J. Dillon, M. Tang, W. C. Carter, and M. P. Harmer, Acta Mater. 55, 6208 (2007).

[3] J. Luo, H. Wang, and Y.-M. Chiang, Journal of the American Ceramic Society 82, 916 (1999).

[4] J. Luo, H. Cheng, K. M. Asl, C. J. Kiely, and M. P. Harmer, Science 333, 1730 (2011).

[5] J. Rickman, H. Chan, M. Harmer, and J. Luo, Surface Science 618, 88 (2013).

[6] J. Rickman, M. Harmer, and H. Chan, Surface Science 651, 1 (2016).

[7] Q. Gao and M. Widom, Phys. Rev. B 90, 144102 (2014).

[8] R. Sun, Z. Wang, M. Saito, N. Shibata, and Y. Ikuhara, Nat Commun 6 (2015).

[9] B. Feng, T. Yokoi, A. Kumamoto, M. Yoshiya, Y. Ikuhara, and N. Shibata, Nat Commun 7 (2016).

[10] Y. Sato, J.-Y. Roh, and Y. Ikuhara, Phys. Rev. B 87, 140101 (2013). 
[11] M. Baram, D. Chatain, and W. D. Kaplan, Science 332, 206 (2011).

[12] M. P. Harmer, Science 332, 182 (2011).

[13] W. Rheinheimer and M. J. Hoffmann, Scripta Materialia 101, 68 (2015).

[14] T. Frolov and Y. Mishin, J. Chem. Phys. 143, 044706 (2015).

[15] M. Tang, W. C. Carter, and R. M. Cannon, Phys. Rev. B 73, 024102 (2006).

[16] M. Tang, W. C. Carter, and R. M. Cannon, Phys. Rev. Lett. 97, 075502 (2006).

[17] J. W. Cahn, J. Physique Colloques 43, 199 (1982).

[18] C. Rottman, J. de Physique Colloque 49, 313 (1988).

[19] E. W. Hart, Grain boundary phase transformations, in Nature and behavior of grain boundaries, edited by H. Hu, pages 155-170, Plenum, New York, 1972.

[20] A. P. Sutton and R. W. Balluffi, Interfaces in Crystalline Materials, Clarendon Press, Oxford, 1995.

[21] Y. Mishin, M. Asta, and J. Li, Acta Mater. 58, 1117 (2010).

[22] T. Frolov, S. V. Divinski, M. Asta, and Y. Mishin, Phys. Rev. Lett. 110, 255502 (2013).

[23] J. J. Hoyt, Modelling and Simulation in Materials Science and Engineering 22, 033001 (2014).

[24] T. Frolov and Y. Mishin, Phys. Rev. B 85, 224107 (2012).

[25] A. Karma, Z. T. Trautt, and Y. Mishin, Phys. Rev. Lett. 109, 095501 (2012).

[26] Z. T. Trautt and Y. Mishin, Acta Mater. 60, 2407 (2012).

[27] T. Frolov and Y. Mishin, Phys. Rev. B 79, 174110 (2009).

[28] A. Suzuki and Y. Mishin, Interface Science 11, 131 (2003).

[29] A. Suzuki and Y. Mishin, Interface Science 11, 425 (2003).

[30] A. Suzuki and Y. Mishin, J. Metastable and Nonocryst. Mater. 19, 1 (2004).

[31] D. L. Olmsted, D. Buta, A. Adland, S. M. Foiles, M. Asta, and A. Karma, Phys. Rev. Lett. 106, 046101 (2011).

[32] S. R. Phillpot and J. M. Rickman, The Journal of Chemical Physics 97, 2651 (1992).

[33] S. R. Phillpot, Phys. Rev. B 49, 7639 (1994).

[34] S. von Alfthan, P. D. Haynes, K. Kashi, and A. P. Sutton, Phys. Rev. Lett. 96, 055505 (2006). 
[35] S. von Alfthan, K. Kaski, and A. P. Sutton, Phys. Rev. B 76, 245317 (2007).

[36] A. L. S. Chua, N. A. Benedek, L. Chen, M. W. Finnis, and A. P. Sutton, Nat Mater 9, 418 (2010).

[37] T. Frolov, D. L. Olmsted, M. Asta, and Y. Mishin, Nat. Commun. 4, 1899 (2013).

[38] T. Frolov, M. Asta, and Y. Mishin, Phys. Rev. B 92, 020103 (2015).

[39] S. V. Divinski, H. Edelhoff, and S. Prokofjev, Phys. Rev. B 85, 144104 (2012).

[40] X.-M. Bai, A. F. Voter, R. G. Hoagland, M. Nastasi, and B. P. Uberuaga, Science 327, 1631 (2010).

[41] M. J. Demkowicz, R. G. Hoagland, and J. P. Hirth, Phys. Rev. Lett. 100, 136102 (2008).

[42] B. P. Uberuaga, L. J. Vernon, E. Martinez, and A. F. Voter, Scientific Reports 5, 9095 EP (2015).

[43] T. LaGrange, K. Arakawa, H. Yasuda, and M. Kumar, Acta Materialia 96, 284 (2015).

[44] P. L. Williams, Y. Mishin, and J. C. Hamilton, Modelling Simul. Mater. Sci. Eng. 14, 817 (2006).

[45] J. W. Cahn, Thermodynamics of solid and fluid surfaces, in Interface Segregation, edited by W. C. Johnson and J. M. Blackely, chapter 1, page 3, American Society of Metals, Metals Park, OH, 1979.

[46] V. A. Ivanov and Y. Mishin, Phys. Rev. B 78, 064106 (2008).

[47] A. Karma, Z. T. Trautt, and Y. Mishin, Phys. Rev. Lett. 109, 095501 (2012).

[48] J. W. Cahn and J. E. Taylor, Acta Mater. 52, 4887 (2004).

[49] A. Suzuki and Y. Mishin, Mater. Sci. Forum 502, 157 (2005).

[50] J. W. Cahn, Y. Mishin, and A. Suzuki, Philos. Mag. 86, 3965 (2006).

[51] J. W. Cahn, Y. Mishin, and A. Suzuki, Acta Mater. 54, 4953 (2006).

[52] D. Molodov, A. Ivanov, and G. Gottstein, Acta Mater. 55, 1843 (2007).

[53] D. A. Molodov and Y. Mishin, Plastic deformation by grain boundary motion: Experiments and simulations, in Microstructural Design of Advanced Engineering Materials, chapter 9, pages 201-233, Wiley, Weinheim, Germany, 2013.

[54] T. Gorkaya, D. A. Molodov, and G. Gottstein, Acta Mater. 57, 5396 (2009).

[55] E. Budke, T. Surholt, S. Prokofjev, L. Shvindlerman, and C. Herzig, Acta Mater. 47, 385 (1999). 
Figure 1: (a) Energies of the $\Sigma 5(210)[001]$ and $\Sigma 5(310)[001]$ GBs in Cu calculated at $0 \mathrm{~K}$ as functions of the the GB density (fraction of atomic plane) [37]. The local energy minima are labeled (b) through (f) and the respective GB structures viewed along the [001] tilt axis are shown in panels (b)-(f). The structural units are outlined in red.

Figure 2: (a) Structural phase transformation in the $\mathrm{Cu} \Sigma 5(310)[001] \mathrm{GB}$ at $800 \mathrm{~K}$ in simulations with an open surface acting as a source of atoms. (b,c) Zoomed-in views of the original split-kite (b) and nucleated filled-kite (c) GB phases

[56] R. Pandit, M. Schick, and M. Wortis, Phys. Rev. B 26, 5112 (1982).

[57] J. Hartford, B. von Sydow, G. Wahnström, and B. I. Lundqvist, Phys. Rev. B 58, 2487 (1998).

[58] D. A. King and D. P. Woodruff, Phase transitions and adsorpate restructuring at metal surfaces, volume 7 of The chemical physics of solid surfaces, Elseview, Amsterdam, Netherlands, 1994.

[59] J. W. Gibbs, On the equilibrium of heterogeneous substances, in The collected works of J. W. Gibbs, volume 1, pages 55-349, Yale University Press, New Haven, 1948.

[60] T. Frolov, Appl. Phys. Lett. 104, 211905 (2014).

[61] W. Yu and M. Demkowicz, Journal of Materials Science 50, 4047 (2015).

[62] J. Han, V. Vitek, and D. J. Srolovitz, Acta Mater. 104, 259 (2016).

Figure 3: GB phase transformations in the $\mathrm{Cu} \Sigma 5(310)[001]$ GB induced by interstitials and vacancies at $800 \mathrm{~K}$ [37]. Panels (a) through (e) show selected GB structures during the cycle involving addition of interstitials and vacancies. 
Figure 4: (a) A bicrystal with split-kite and filled-kite GB phases of the $\Sigma 5(210)[001]$ GB in $\mathrm{Cu}(\mathrm{Ag})$ alloy connected to open surfaces [37]. Zoomed-in views of the split-kite (b) and filled-kite (c) structures reveal bilayer and monolayer segregation patterns, respectively. (d,f) Segregation isotherms calculated for the two phases at 900K. (e) GB free energies relative to the phase coexistence state with $c *=0.02 \%$. (g) Schematic of the phase coexistence line $c_{*}(T)[38]$.

Figure 5: (a,b) Bicrystals with the split-kite (a) and filled-kite (b) phases of the $\mathrm{Cu}$ $\Sigma 5(210)[001] \mathrm{GB}$ after $30 \mathrm{~ns}$ of stress-driven motion at $700 \mathrm{~K}$ [60]. The initially vertical red stripe is a marker line illustrating the shear deformation. The dashed white line shows the initial GB position and the black arrow the final position. (c) Inverse coupling factor as a function of temperature. The horizontal lines represent the ideal coupling factors for the $<100>$ and $<110>$ coupling modes. (d) Average shear stress during the coupled GB motion as a function of temperature. The vertical dashed line indicates the bulk melting point.

Figure 6: Arrhenius diagrams of GB diffusion flux $P$ for $\mathrm{Cu}$ self-diffusion (lower curves) and Ag impurity diffusion (upper curves) in the kite and split-kite phases of the $\Sigma 5(310)[001]$ GB. For Ag diffusion, the experimental data [39] are shown for comparison. Diffusion parallel and normal to the tilt axis is represented by filled and open symbols, respectively. 


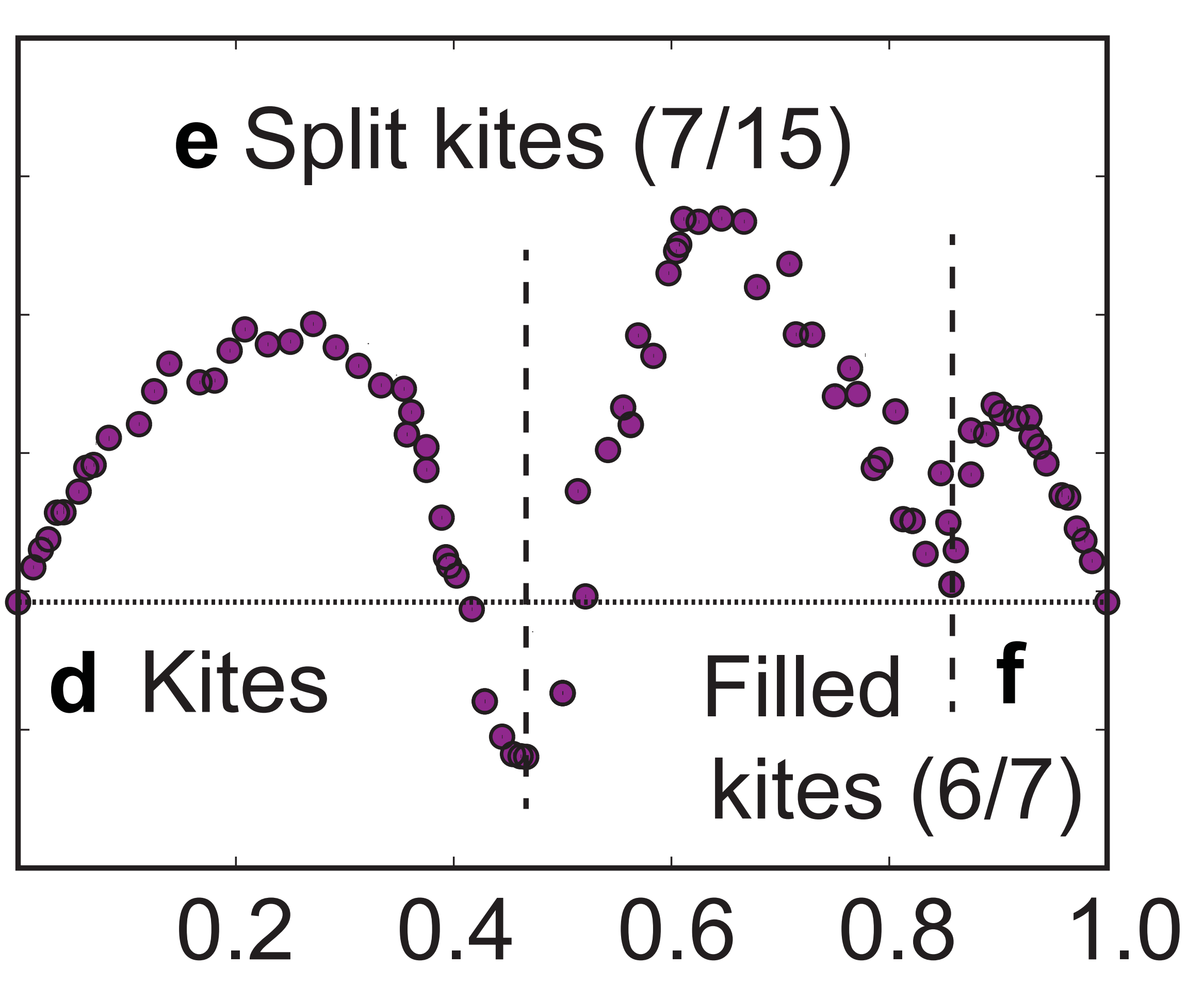

Fraction of (210) plane

(c)

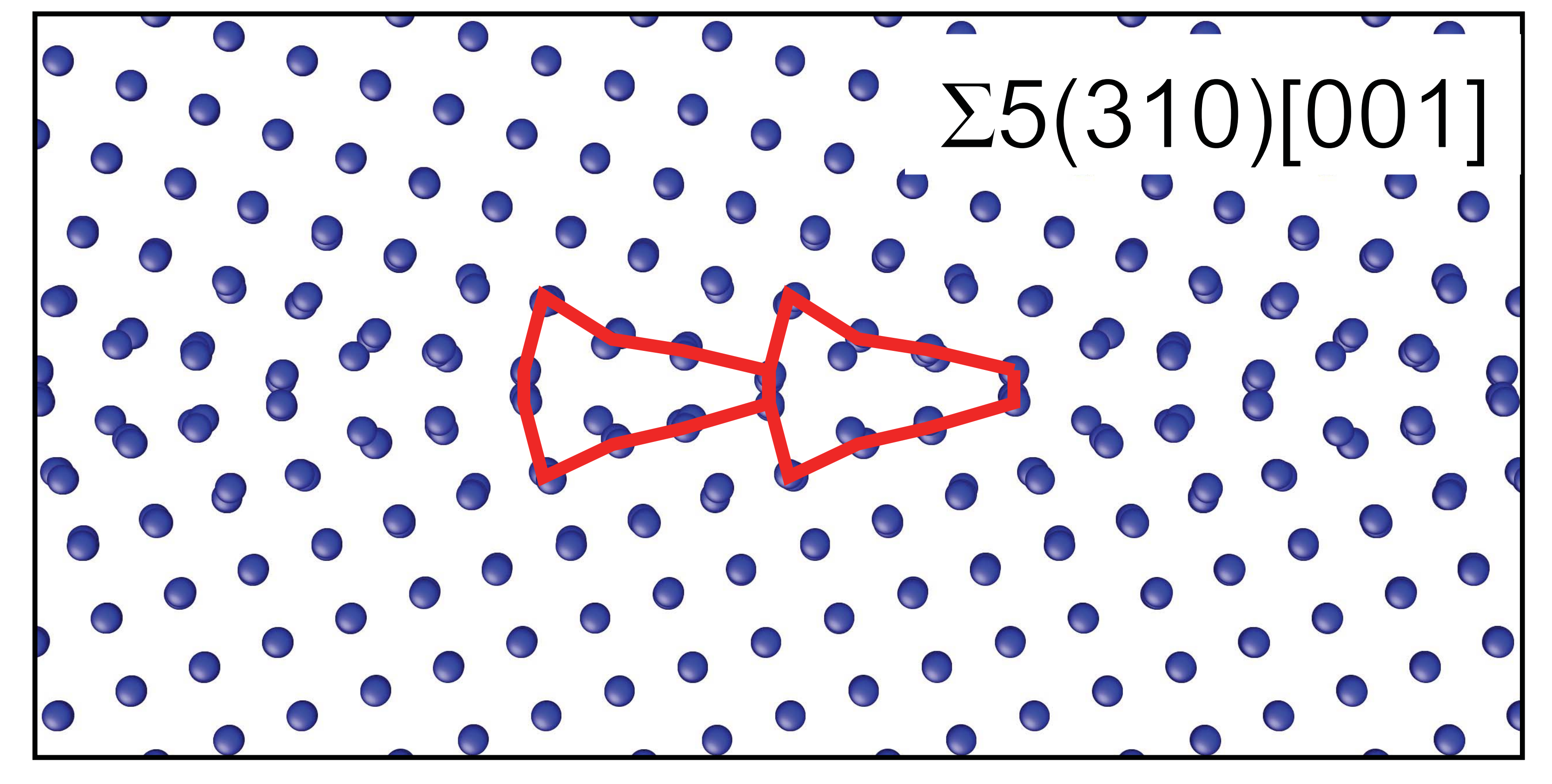

(d)

(b)

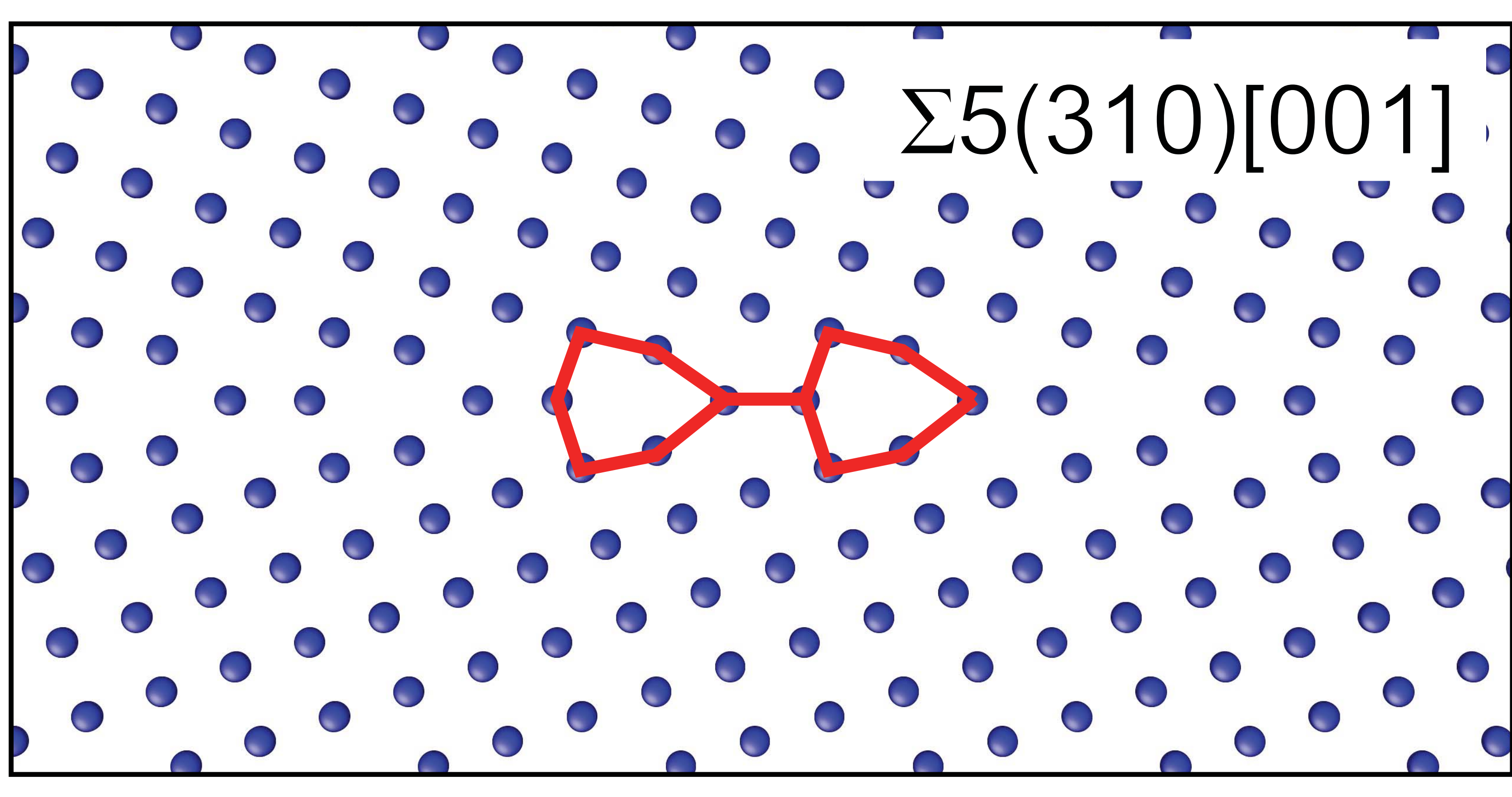

(e)

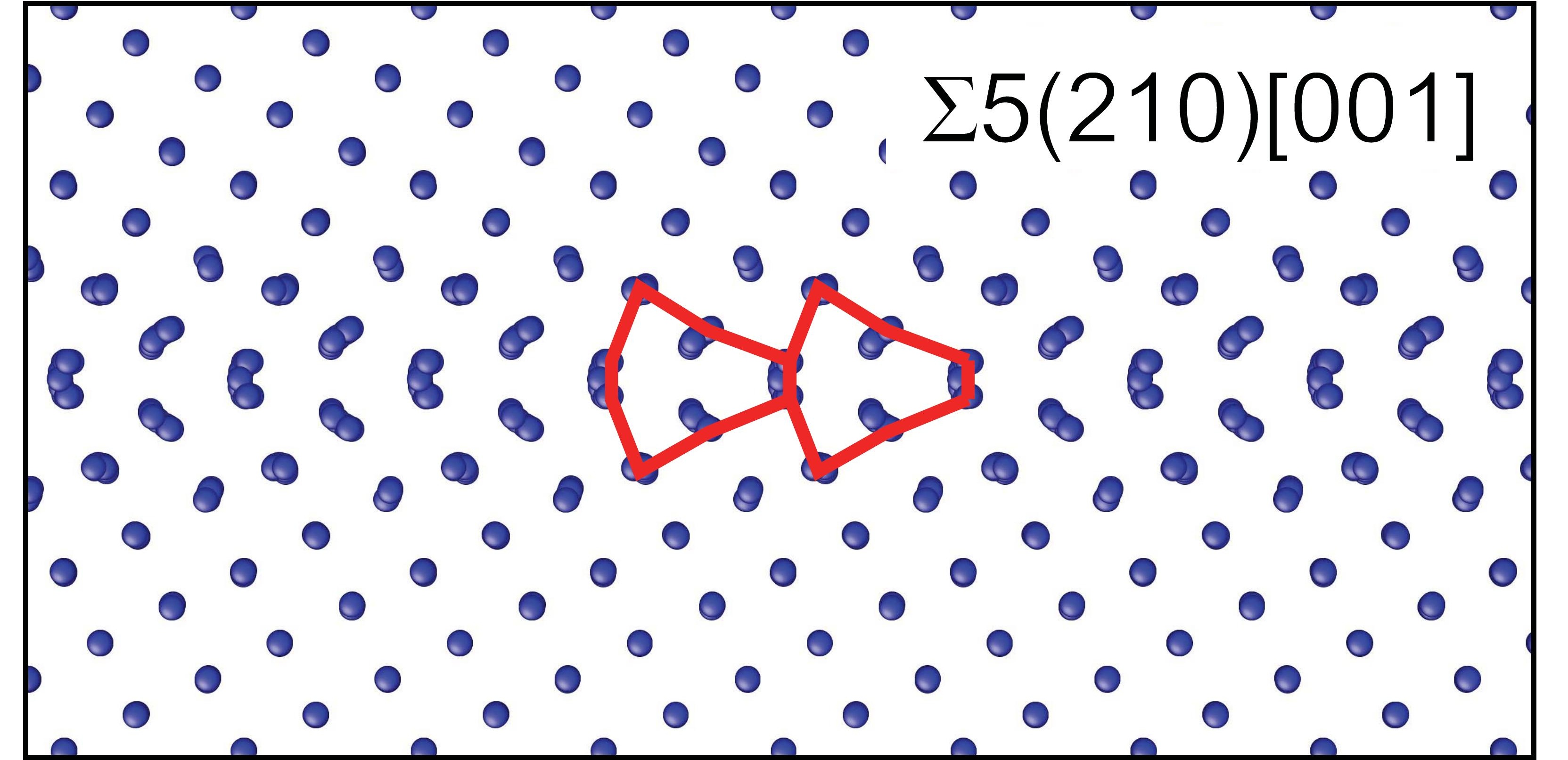

(f)

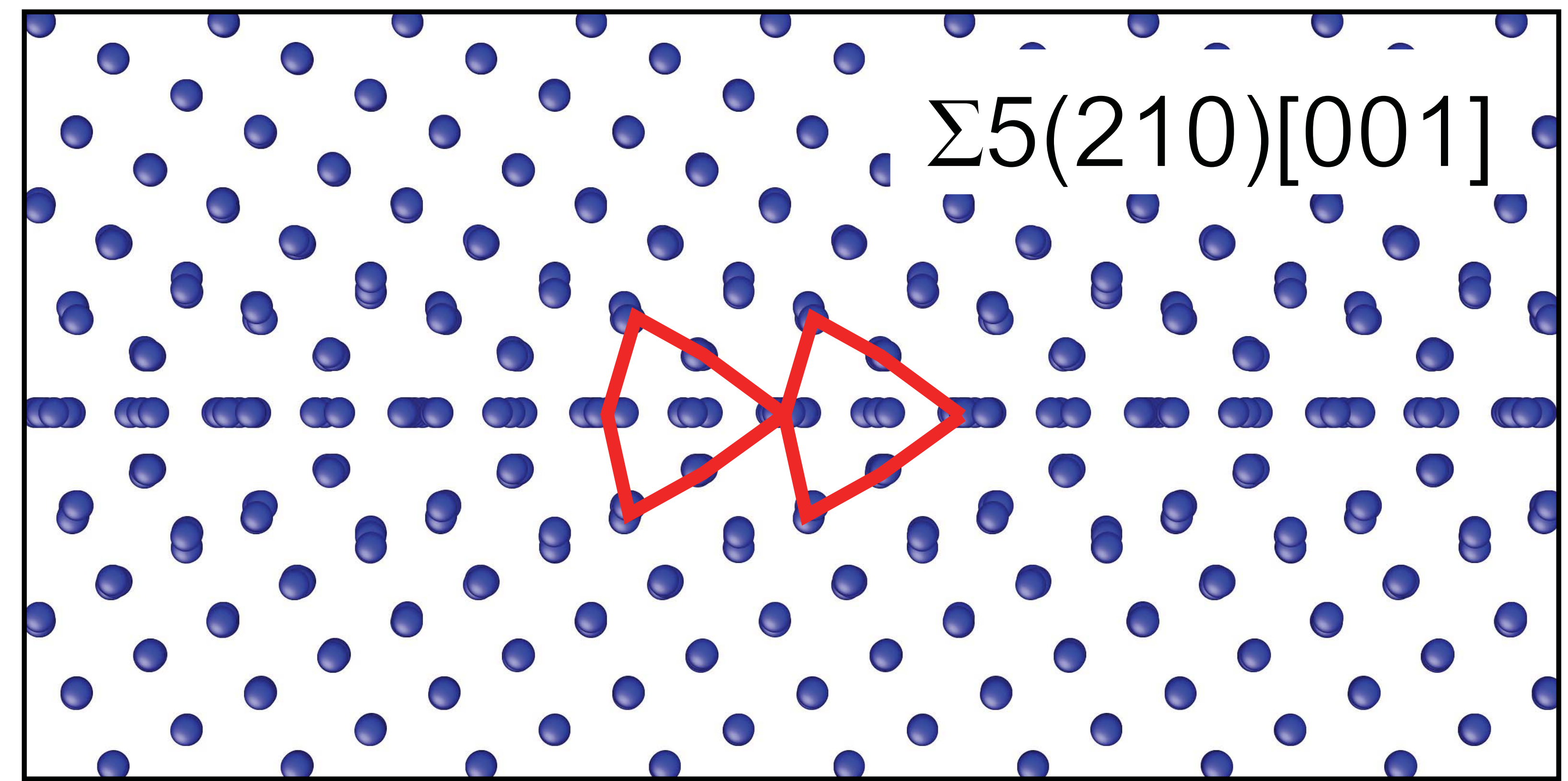


(a)

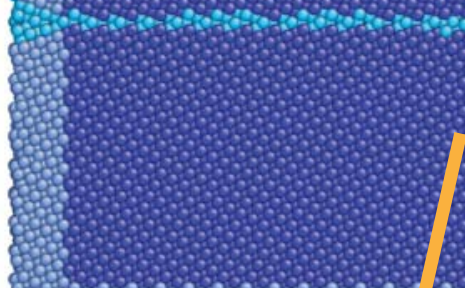

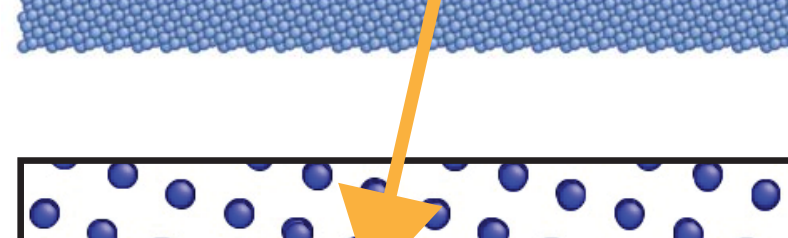

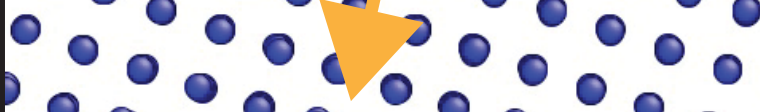

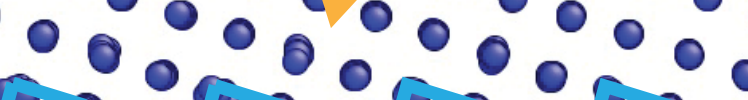

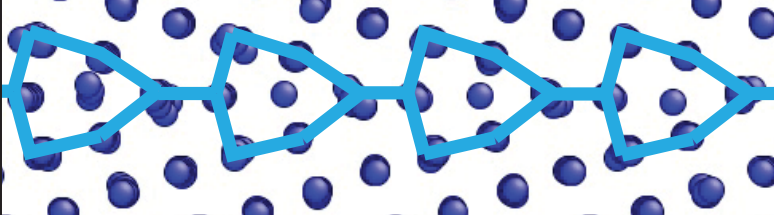
(b)

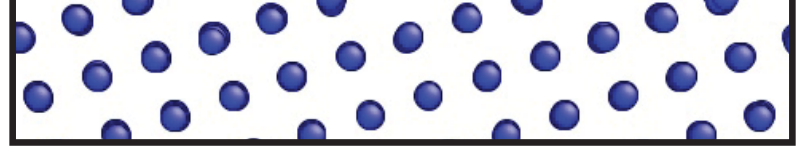


(a)

(b)

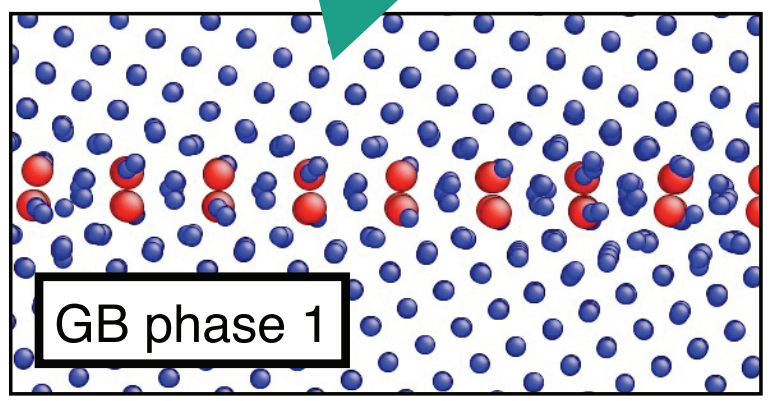

(d)

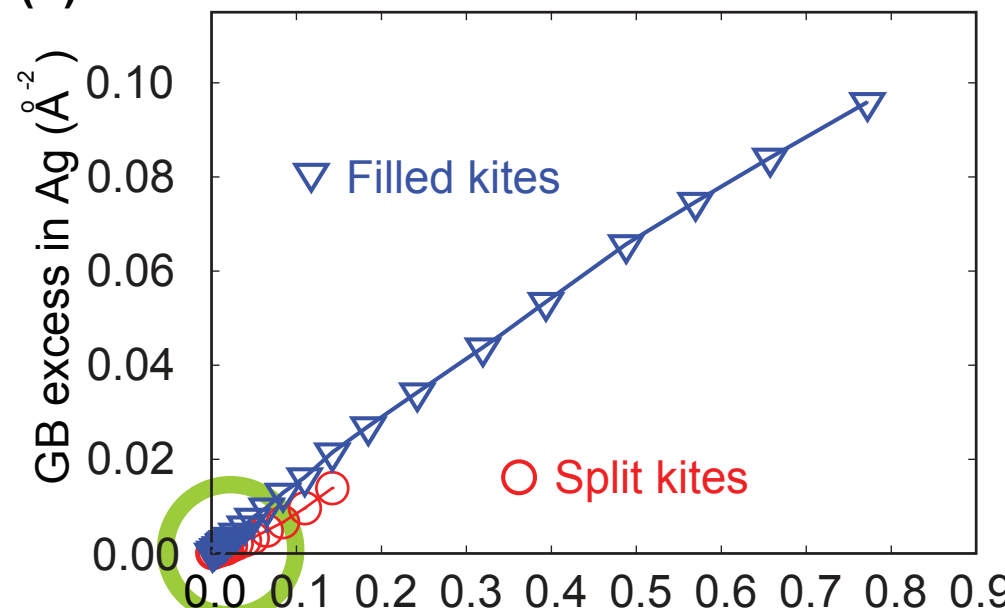

(f)

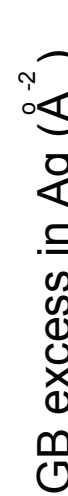

0.000

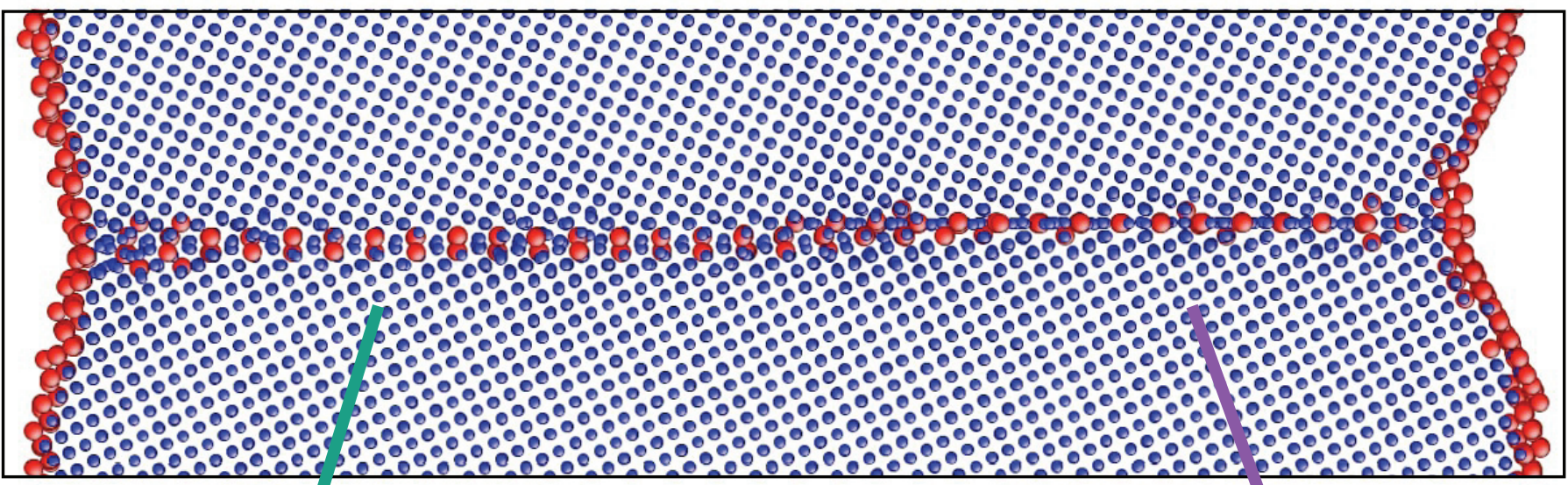

(c)

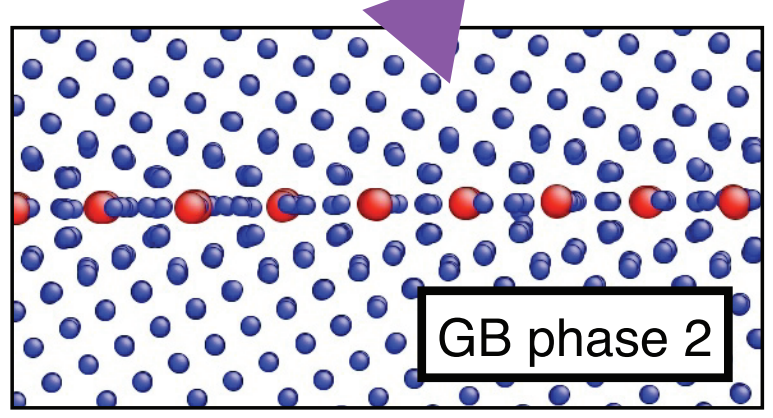

(e)

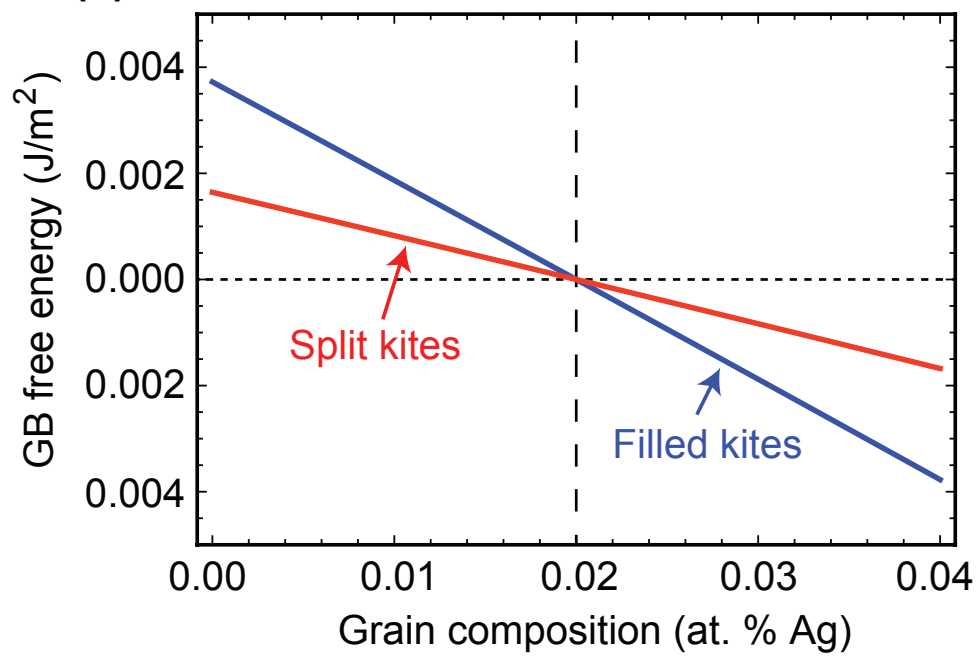

(g)

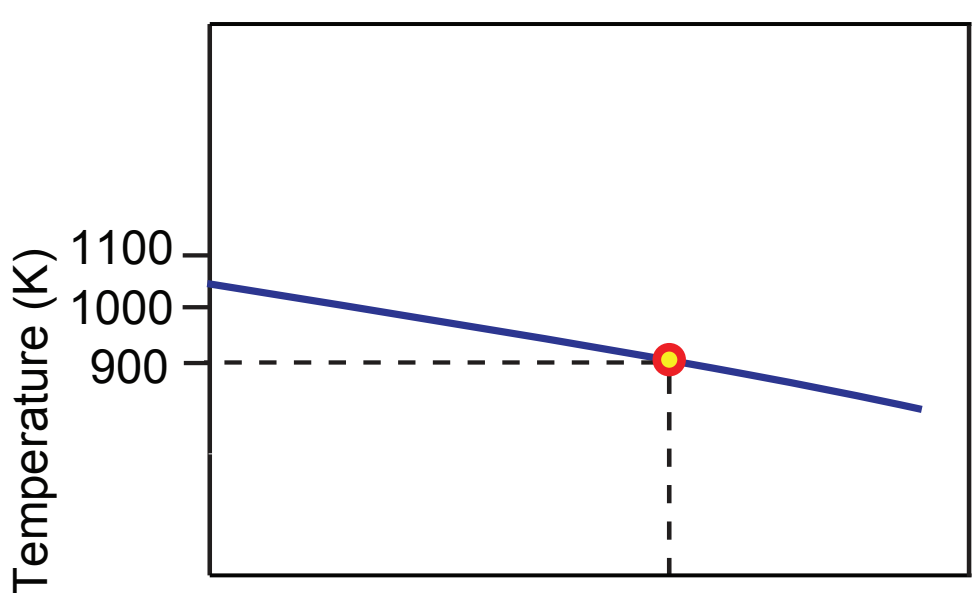

0

0.02

Grain composition (at. \% Ag) 


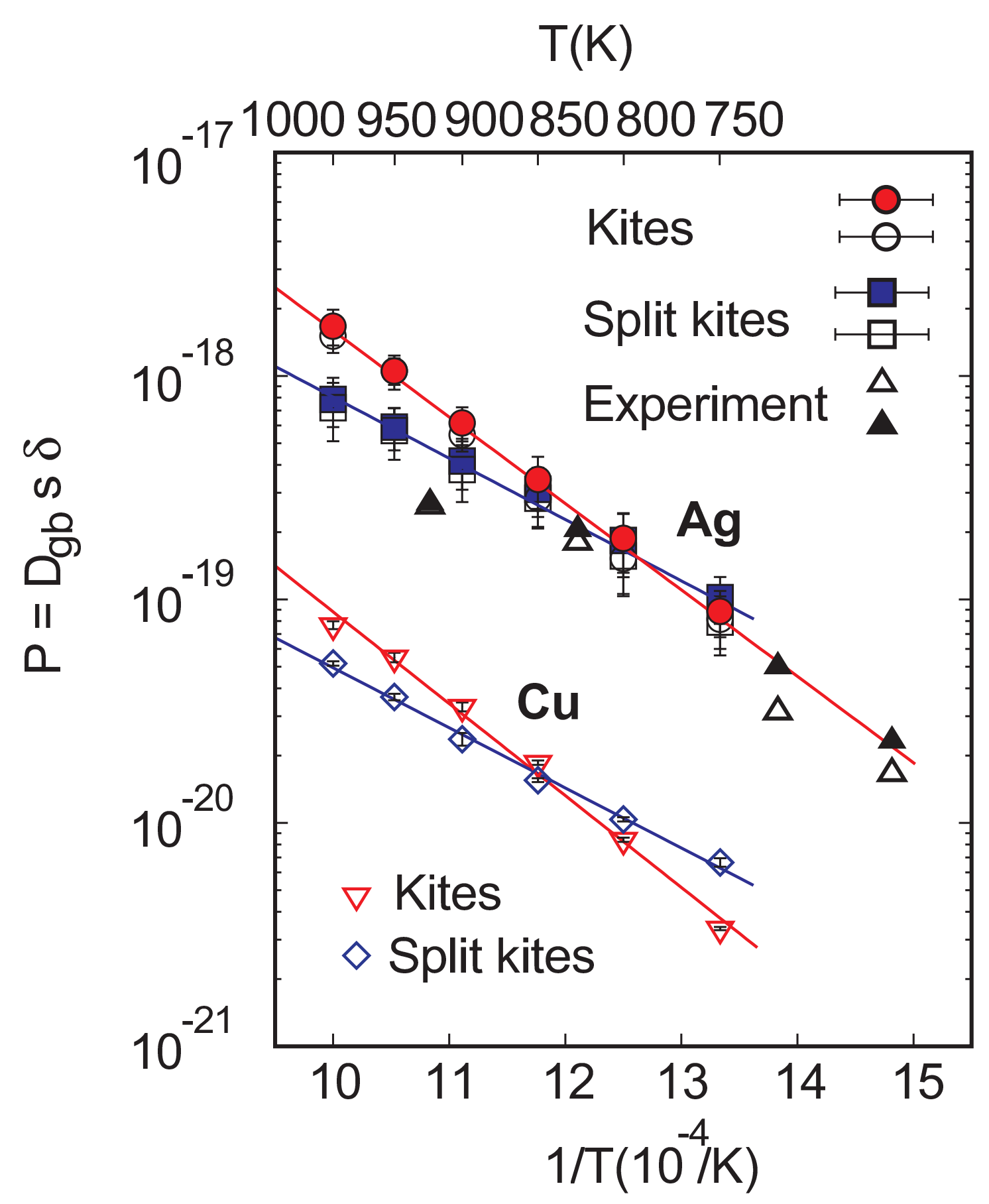

